\title{
A PRECISION MEASUREMENT OF THE BRANCHING
}

RATIO $K^{+} \rightarrow \pi^{+} \pi^{0} / K^{+} \rightarrow \mu^{+} \nu_{\mu}^{*}$

\author{
T. Usher, ${ }^{\star}$ M. Fero, ${ }^{\star}$ M. Gee, N. A. Graf, ${ }^{\circ}$ M. Mandelkern, \\ D. Schultz ${ }^{\star}$ and J. Schultz \\ Stanford Linear Accelerator Center, \\ Stanford University, Stanford, CA 94309 \\ and \\ Department of Physics, University of California, \\ Irvine, CA 92717, USA
}

\begin{abstract}
A measurement of the branching ratio $K^{+} \rightarrow \pi^{+} \pi^{0} / K^{+} \rightarrow \mu^{+} \nu_{\mu}$ was made using stopped kaons from $p \bar{p}$ annihilations at rest and a magnetic spectrometer to measure the momenta of the charged decay product to the $1 \%$ to $1.5 \%$ level. The determination is based on 45,500 events passing final data cuts. The resulting ratio is $.3329 \pm .0047$ (statistical) \pm .0010 (systematic).
\end{abstract}

Submitted to Physical Review D.

${ }^{*}$ Work supported by Department of Energy contract DE-AC03-76SF00515.

${ }^{\circ}$ Present address: Fermi National Laboratory, Batavia, IL 60510.

${ }^{\star}$ Present address: Stanford Linear Accelerator Center, Stanford University,

Stanford, CA 94309. 


\section{INTRODUCTION}

We have made a measurement of the ratio between the two principal decay modes of charged kaons, the non-leptonic two pion decay, and the semi-leptonic decay into a muon and neutrino. The ratio has been measured previously with high statistics [1-4]. The present measurement is made with a technique distinctly different from any of the previous measurements, and with a momentum resolution and background level superior to that of any of the previous experiments.

The semileptonic mode $K \rightarrow \mu \nu_{\mu}$ is understood in terms of Cabibbo theory and is chararacterized by $f_{k}$, the matrix element of the axial vector current for the kaon. The hadronic mode $K \rightarrow \pi \pi$ requires the explicit computation of a hadronic matrix element which, as yet, has not been performed (e.g., see Ref. [5]). While theory is currently unable to confront a measurement as precise as we are reporting here, one must be hopeful that as QCD calculations improve, fundamental observables such as this branching ratio will become calculable.

The measurement of this branching ratio was made as part of Experiment PS183, a LEAR (CERN) experiment designed to study the spectra of charged particles and of gamma rays resulting from proton-antiproton annihilations at rest in hydrogen and deuterium.

\section{APPARATUS}

\section{A. Detector}

Experiment PS183 consisted of a large, well-instrumented magnetic spectrometer.designed to momentum analyze single charged particles or $e^{+} e^{-}$pair conversions of gamma rays resulting from the annihilation of protons with antiprotons. It has 
been described extensively in several previous publications [6,7], most recently in great detail in Ref. [8]. Relevant to the data presented here are: (a) the presence of a beam counter ( $\mathrm{S} 2$ ) to signal antiprotons entering the $70-\mathrm{cm}$ long by $7-\mathrm{cm}$ diameter liquid hydrogen target; (b) two sets of planar drift chambers (RDC, with $x, u$ and $v$ planes) located between the liquid hydrogen target and the magnet; (c) a $0.5-\mathrm{cm}$ thickplastic scintillation counter $(Q)$ to signal charged particles entcring the magnet aperture; (d) three multiwire proportional chambers (MWPCs) at the entrance to the magnetic volume, B ( $x$ plane), $\mathrm{C} 1$ ( $x$ and $y$ planes), C2 ( $x$ and $y$ planes); (e) and two sets of two MWPCs-D1 ( $x$ plane) and D2 ( $x$ and $y$ planes) located to the upstream side of the magnetic volume, and similarly E1 and E2 located to the downstream side of the magnetic volume. In addition, decay products from kaons stopping in the target region passed through a total of $1.25 \mathrm{~cm}$ of Dallite (used to form walls for the target vacuum and a cylindrical MWPC which surrounded the target), two additional $0.5-\mathrm{cm}$ thick plastic scintillators and a $0.06-\mathrm{cm}$ thick lead foil (the gamma ray converter) before completing the trigger. For the data presented here, the magnet was operated at field strengths of $7.5 \mathrm{kG}$, giving an approximate $1 \%$ momentum resolution (rms) in the vicinity of the lines of interest (180 to $240 \mathrm{MeV} / \mathrm{c}$ ) and $3.5 \mathrm{kG}$ with an approximate $1.5 \%$ resolution.

\section{B. Trigger}

The data sample discussed here is based upon events with a single charged track entering the spectrometer and turning to the upstream or downstream side of the magnetic volume. The trigger for this topology was a coincidence including the beam counter (S2), the $\mathrm{Q}$ counter at the entrance to the magnet aperture and one-of the counters $(\mathrm{D}, \mathrm{E})$ shadowed by the D or E set of MWPCs. During normal running designed to study antiproton annihilations, $K$ decays were overwhelmed by 
annihilation products. For the $K$ decay studies an additional trigger requirement was imposed that required charged tracks passing through the $\mathrm{Q}$ counter to be $2 \mathrm{~ns}$ or more later than those from antiproton annihilations.

\section{DATA ANALYSIS}

\section{A. Data sample}

Five distinct runs were performed with the above kaon decay trigger: at $\pm 3.5 \mathrm{kG}$ in May 1985 with an incident antiproton beam momentum of $330 \mathrm{MeV} / \mathrm{c}$, at $-7.5 \mathrm{kG}$ in July 1986 with an incident antiproton beam momentum of $430 \mathrm{MeV} / \mathrm{c}$, and at $\pm 7.5 \mathrm{kG}$ in August 1986 with an incident antiproton beam momentum of $350 \mathrm{MeV} / \mathrm{c}$. Each of these runs recorded from 140,000 to 240,000 triggers.

\section{B. Analysis}

Analysis of the data proceeded in two steps. The first involved the determination of the momentum of single charged tracks through the use of a modified circle fit procedure utilizing the $x$-coordinates from the B, C2 and D2 (or E2 depending upon charge) wire chambers located inside the magnet volume. The total numbers of events reconstructing (of both charges) are summarized in the "First Pass" column of Table I, with the number of positive charge particles then summarized in the "Positive Charge" column. Figure 1 provides an example of the resulting spectrum. In the next step, these reconstructed tracks were passed through a series of cuts designed to reduce background and eliminate "bad" tracks. In order of application, these were: 
- a cut on the pulse height of the $\mathrm{Q}$ counter designed to remove low-momentum protons $(<300 \mathrm{MeV} / \mathrm{c}$ ) ejected from the target region by annihilation pions ("No Protons" cut),

- fiducial cuts on the B, C1, C2, D1 and D2 (or E1 and E2 depending upon field polarity) $x$ - and $y$-coordinates in order to eliminate tracks passing through the G-10 wire chamber frames ("Wire Cuts" cut),

- matching of wire chamber tracks to those reconstructed in the RDC drift chambers ("RDC Track" cut),

- a cut on the projection of the track reconstructed in the RDC drift chambers to the centerline of the target axis to limit decays to those kaons stopping in the target region ("Vertex" cut), and

- a cut on the pulse height of the beam counter to eliminate events in which two or more antiprotons entered the target during the trigger live time (hence removing events possibly occuring from the annihilation of late secondary antiprotons) ("Beam Pulse" cut).

The effects of these cuts are summarized in Table I. The variation in the effect of the proton cut is due to the different beam momenta of the various runs, as well as the slightly different target configurations between 1985 and 1986 . The vertex cut is sensitive to the upstream-downstream position of the antiproton stopping distribution relative to the centerline of the spectrometer. Finally, the beam counter pulse height information was unavailable for part of the May $1985+3.5 \mathrm{kG}$ data run, this cut is not_applied to the affected data. 
The resulting spectra were then simulteneously fit to two peaks (using a skewed- Gaussian form for each peak) and a smooth background (a fifth degree polynomial) in the region 100 to $300 \mathrm{MeV} / \mathrm{c}$. Skewed-Gaussian forms were used to account for the energy loss Landau tails in the peaks. A polynomial was chosen to represent the background mostly because a good model for it did not exist and because it was smooth and nearly flat (a good model did not exist since while the background was partially composed of multi-body kaon decay products, it was mostly made up of "broken" events from antiproton-proton annihilations which the data analysis cuts had failed to completely remove). Figures 2 and 3 provide examples of the fits for the $3.5 \mathrm{kG}$ and $7.5 \mathrm{kG}$ data runs; the chi-square per degree of freedom for each fit is given in column 3 of Table II. The number of events in the two peaks were then detcrmined from a numerical integration of the fit results with the errors on the number of events in the two peaks determined from a numerical evaluation of the full covariance matrix (including contributions from the polynomial background). Column 2 of Table II summarizes the results of this procedure for the five runs. Column 4 of Table II then presents the ratios of the two decay modes for the five analyzed runs, uncorrected for systematic errors.

\section{Correction for systematic effects}

The ratios determined from the above data analysis were subject to several sources of systematic effects:

o differing geometrical acceptances at the momenta of the two peaks,

- _. differing inflight decay losses of pions versus muons at these momenta, 
- losses of pions due to hadronic interactions with the materials comprising the spectrometer, and

- losses of events in the peak regions from large angle scatters or much more than average energy losses.

In order to account for these effects, and therefore obtain an accurate ratio between the two kaon decay modes, a Monte Carlo simulation of the decays of positively charged kaons generated from antiproton-proton annihilations was performed. This consisted of (1) modeling the antiproton annihilation vertex distribution, (2) generating and tracking low momentum kaons to their stop points, (3) tracking the charged decay product through a GEANT (version 3.10) simulation of the spectrometer, (4) digitizing the track information, and (5) outputting to tape in the form of the original raw data tapes. This procedure was performed seperately for each of the decay modes of interest (once for all kaons decaying into $\pi^{+} \pi^{0}$, and once with all decaying into $\mu^{+} \nu_{\mu}$ ) with enough initial events generated to insure small statistical errors with respect to the data. The resulting Monte Carlo data tapes were then passed through the regular data analysis program described above to produce two sets of Monte Carlo raw spectra which were then added together and fit to the same functional form as the raw data. The systematic correction factor was then determined as the ratio of the number of events in the muon peak to the number of cvents in the pion peak (the inverse of the data ratio). Finally, radiative decay losses were taken into effect by folding in the calculated contribution from $K^{+} \rightarrow \mu^{+} \nu_{\mu} \gamma$ (the corresponding radiative decay for pions was negligible).

- During it's development, the Monte Carlo was constantly checked against the data for consistency. The most critical check was in comparing the fit values of 
the Monte Carlo peaks against those of the raw data. For the $7.5 \mathrm{kG}$ data, the centers of the Monte Carlo peaks were shifted an average $.3 \mathrm{MeV} / \mathrm{c}$ higher in momentum than the raw data peaks, were an average $.1 \mathrm{MeV} / \mathrm{c}$ narrower and were more skewed towards lower momenta, all as expected given the Landau Distribution used by GEANT to model the energy loss. As discussed below, these discrepencies were used to obtain an estimate for the systematic error.

The above procedure was performed seperately for each of the five distinct data runs (to account for different magnetic field polarities and changes to the target configuration to reflect the different incident antiproton beam momenta). The resulting five correction factors are presented in column 5 of Table II.

\section{Estimation of systematic error}

The two dominant contributions to the systematic error on the correction factor were found to be from the uncertainty in the ratio of the geometrical acceptances at the two peak momenta, and from the uncertainty in the correction for loss of pions due to hadronic interactions.

For the same kaon stopping distribution, the acceptance correction factor at the two peak momenta depended most critically upon the uncertainty in the $x$-wire coordinates of the MWPCs. To obtain an upper limit to the systematic error this could introduce, each chamber individually was moved one-half wire spacing $(.15 \mathrm{~cm})$ and a new acceptance correction factor determined. Averaging overall acceptance correction factors thus obtained led to an upper limit of $0.21 \%$ in the systematic uncertainty of the acceptance correction factor. 
The uncertainty in the correction for loss of pions due to hadronic interactions consisted of two parts, that from the uncertaintities in the hadronic cross sections for the materials traversed at the nominal pion energy and that from the uncertainty in the amount of material present in the spectrometer. The contribution of the formcr was determined by calculating the error in the hadron correction factor, based upon published errors in the cross sections for the four materials contributing most significantly to pion loss (scintillator, lead, dallite and hydrogen), and was found to be $0.16 \%$. An estimate of the uncertainty in the amount of material present in the spectrometer was determined by comparing the difference in mean energy loss predicted by the Monte Carlo and observed in the data. Sincc scintillator represented the single largest contributor to the hadronic losses in this experiment, an upper limit to the correction factor was obtained by assuming this represented the "extra" material and was found to be $0.11 \%$.

Combining the above uncertaintities gave an estimate to the systematic error of $0.29 \%$.

\section{FINAL RESULTS}

Column 6 of Table II presents the final corrected $K^{+} \rightarrow \pi^{+} \pi^{0} / K^{+} \rightarrow \mu^{+} \nu_{\mu}$ ratio for each of the five data sets used in this analysis. The final ratio determined by this experiment is the weighted average of these five numbers: $.3329 \pm .0047$ (statistical) \pm .0010 (systematic).

Table III compares the result of this experiment to the four previous cxpcrimental results (it should be noted that the experiments in Refs. [1-3] represent direct measurements of the ratio while the result of Ref. [4] is determined from thcir 
measurement of the absolute branching ratios). The result of this experiment agrees well with the previous results.

The good agreement of the result of this experiment with the previous experiments, given the substantially different experimental technique employed, adds further confidence in the accuracy to which the ratio $K^{+} \rightarrow \pi^{+} \pi^{0} / K^{+} \rightarrow \mu^{+} \nu_{\mu}$ has been determined. The different technique employed also offers several advantages of this result over those of the previous experiments. Chief amongst these was the use of proton-antiproton annihilations at rest to provide a high quality statistics sample of clean, easily identifiable, kaon decays (as opposed to the use of secondary beams). The Experiment PS183 spectrometer itself was of a straightforward design and the systematics were well understood. Finally, the spectrometer had superior momentum resolution compared to the previous experiments, enabling clean separation of the peaks in the resulting kaon decay spectrum.

\section{ACKNOWLEDGMENT}

This work was supported in part by the US National Science Foundation, and by the US Department of Energy, contract DE-AT03-76ER71019-TASKB. 


\section{REFERENCES}

1. L. B. Auerbach et al., Phys. Rev. 155, 1505 (1967).

2. M. E. Zeller et al., Phys. Rev. 182, 1420 (1969).

3. I-H. Chiang et al., Phys. Rev. 6D, 1254 (1972).

4. A. O. Weissenberg et al., Nucl. Phys. B115, 55 (1976).

5. M. B. Gavela et al., "The Kaon $B$-parameter and $K-\pi$ and $K-\pi \pi$ Transition Amplitudes on the Lattice," CERN TH-4905/87 (November 1987).

6. A. Angelopoulos et al., Phys. Lett. B159, 210 (1985).

7. A. Angelopoulos et al., Phys. Lett. B178, 441 (1985).

8. N. A. Graf et al., Phys. Rev. D B159, 210 (1985). 


\section{TABLE CAPTIONS}

I. Data analysis event selection summary. The percentages represent the fraction of events surviving the previous cut.

II. Data analysis fits and results.

III. Comparison to previous experiments. 
Table I. Data analysis event selection summary. The percentages represent the fraction of events surviving the previous cut.

\begin{tabular}{|c|c|c|c|c|c|c|c|c|}
\hline Data Set & $\begin{array}{c}\text { Raw } \\
\text { Events }\end{array}$ & $\begin{array}{l}\text { First } \\
\text { Pass }\end{array}$ & $\begin{array}{l}\text { Positive } \\
\text { Charge }\end{array}$ & $\begin{array}{c}\text { No } \\
\text { Protons }\end{array}$ & $\begin{array}{l}\text { Wire } \\
\text { Cuts }\end{array}$ & $\begin{array}{l}\text { RDC } \\
\text { Track }\end{array}$ & Vertex & $\begin{array}{l}\text { Beam } \\
\text { Pulse }\end{array}$ \\
\hline May +3.5 & 198082 & $\begin{array}{l}99321 \\
50.1 \%\end{array}$ & 55406 & $\begin{array}{l}50285 \\
90.8 \%\end{array}$ & $\begin{array}{l}41129 \\
81.8 \%\end{array}$ & $\begin{array}{l}32111 \\
78.1 \%\end{array}$ & $\begin{array}{l}15993 \\
49.6 \%\end{array}$ & $\begin{array}{l}12486 \\
78.4 \%\end{array}$ \\
\hline May -3.5 & 164269 & $\begin{array}{l}82935 \\
50.5 \%\end{array}$ & 44607 & $\begin{array}{l}41184 \\
92.3 \%\end{array}$ & $\begin{array}{l}34272 \\
83.2 \%\end{array}$ & $\begin{array}{l}26304 \\
76.8 \%\end{array}$ & $\begin{array}{l}13795 \\
52.4 \%\end{array}$ & $\begin{array}{r}9273 \\
67.2 \%\end{array}$ \\
\hline Jul -7.5 & 246485 & $\begin{array}{r}152812 \\
62.0 \%\end{array}$ & 96092 & $\begin{array}{l}66560 \\
69.3 \%\end{array}$ & $\begin{array}{l}57484 \\
86.4 \%\end{array}$ & $\begin{array}{l}43296 \\
75.3 \%\end{array}$ & $\begin{array}{l}20568 \\
47.5 \%\end{array}$ & $\begin{array}{l}14296 \\
69.5 \%\end{array}$ \\
\hline Aug +7.5 & 144196 & $\begin{array}{l}95788 \\
66.4 \%\end{array}$ & 58657 & $\begin{array}{l}41925 \\
71.5 \%\end{array}$ & $\begin{array}{l}35661 \\
85.1 \%\end{array}$ & $\begin{array}{l}29067 \\
81.5 \%\end{array}$ & $\begin{array}{l}17892 \\
61.6 \%\end{array}$ & $\begin{array}{l}12724 \\
71.1 \%\end{array}$ \\
\hline Aug -7.5 & 236419 & $\begin{array}{r}152296 \\
64.4 \%\end{array}$ & 94024 & $\begin{array}{l}81854 \\
87.1 \%\end{array}$ & $\begin{array}{l}70366 \\
86.0 \%\end{array}$ & $\begin{array}{l}54261 \\
77.1 \%\end{array}$ & $\begin{array}{l}34456 \\
63.5 \%\end{array}$ & $\begin{array}{l}23976 \\
69.6 \%\end{array}$ \\
\hline
\end{tabular}


Table II. Data analysis fits and results.

\begin{tabular}{|lccccc|}
\hline Peak & Area & $\chi^{2} /$ dof & $\begin{array}{c}\text { Uncorrected } \\
\text { Ratio }\end{array}$ & Correction & $\begin{array}{c}\text { Corrected } \\
\text { Ratio }\end{array}$ \\
\hline \hline $5 / 85+3.5 \pi$ & $1345 \pm 53$ & & & & \\
$5 / 85+3.5 \mu$ & $4682 \pm 74$ & 1.02 & $.2873 \pm .0122$ & $1.179 \pm .021$ & $.3387 \pm .0156$ \\
$5 / 85-3.5 \pi$ & $1166 \pm 38$ & & & & \\
$5 / 85-3.5 \mu$ & $4122 \pm 82$ & 1.25 & $.2829 \pm .0108$ & $1.176 \pm .021$ & $.3327 \pm .0140$ \\
& & & & & \\
$7 / 86-7.5 \pi$ & $1745 \pm 47$ & & & & \\
$7 / 86-7.5 \mu$ & $7188 \pm 87$ & 1.01 & $.2428 \pm .0072$ & $1.3797 \pm .0167$ & $.3350 \pm .0107$ \\
& & & & & \\
$8 / 86+7.5 \pi$ & $1676 \pm 44$ & & & & \\
$8 / 86+7.5 \mu$ & $6842 \pm 84$ & 0.94 & $.2450 \pm .0071$ & $1.3839 \pm .0172$ & \\
$8 / 86-7.5 \pi$ & $3246 \pm 60$ & & & & $.3279 \pm .0072$ \\
$8 / 86-7.5 \mu$ & $13688 \pm 118$ & 1.23 & $.2371 \pm .0048$ & $1.3830 \pm .0118$ & \\
\hline
\end{tabular}


Table III. Comparison to previous experiments.

\begin{tabular}{|lcc|}
\hline Experiment & Reference & Ratio \\
\hline \hline L. B. Auerbach et al. & 1 & $.3277 \pm .0065$ \\
M. E. Zeller et al. & 2 & $.305 \pm .018$ \\
I-H. Chiang et al. & 3 & $.3349 \pm .0050$ \\
A. O. Weissenberg et al. & 4 & $.3355 \pm .0057$ \\
This experiment & & $.3329 \pm .0047$ \\
\hline
\end{tabular}




\section{FIGURE CAPTIONS}

1. Example of the raw spectrum produced with the additional trigger requirement that the $Q$ scintillation counter at the entrance to the spectrometer fire "late" by at least two nanoseconds. The spectrum is from the August $1986-7.5 \mathrm{kG}$ run.

2. Final fitted spectrum from the August $1986-7.5 \mathrm{kG}$ run.

3. Final fitted spectrum from the May $1985+3.5 \mathrm{kG}$ run. 


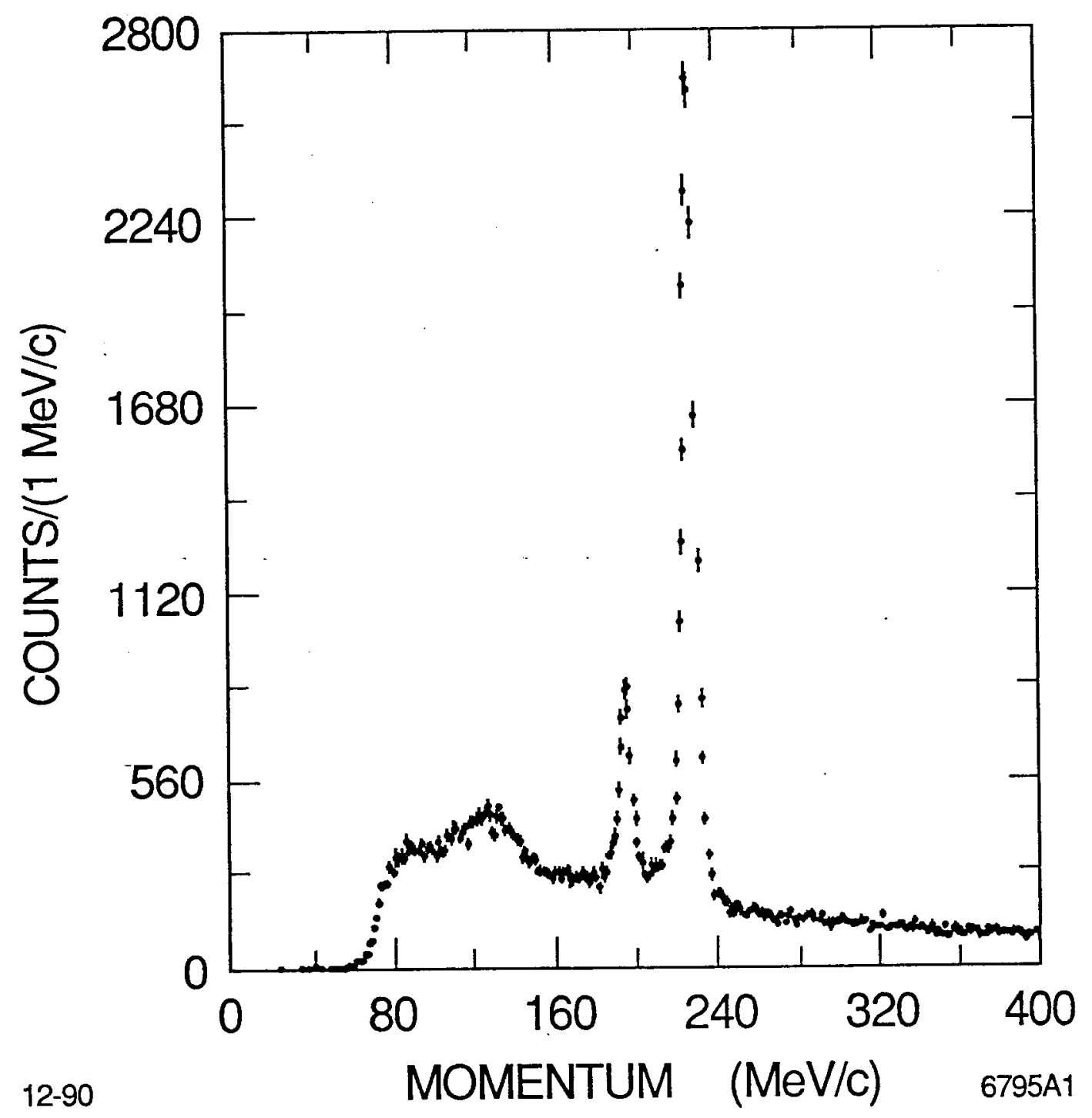

Fig. 1 


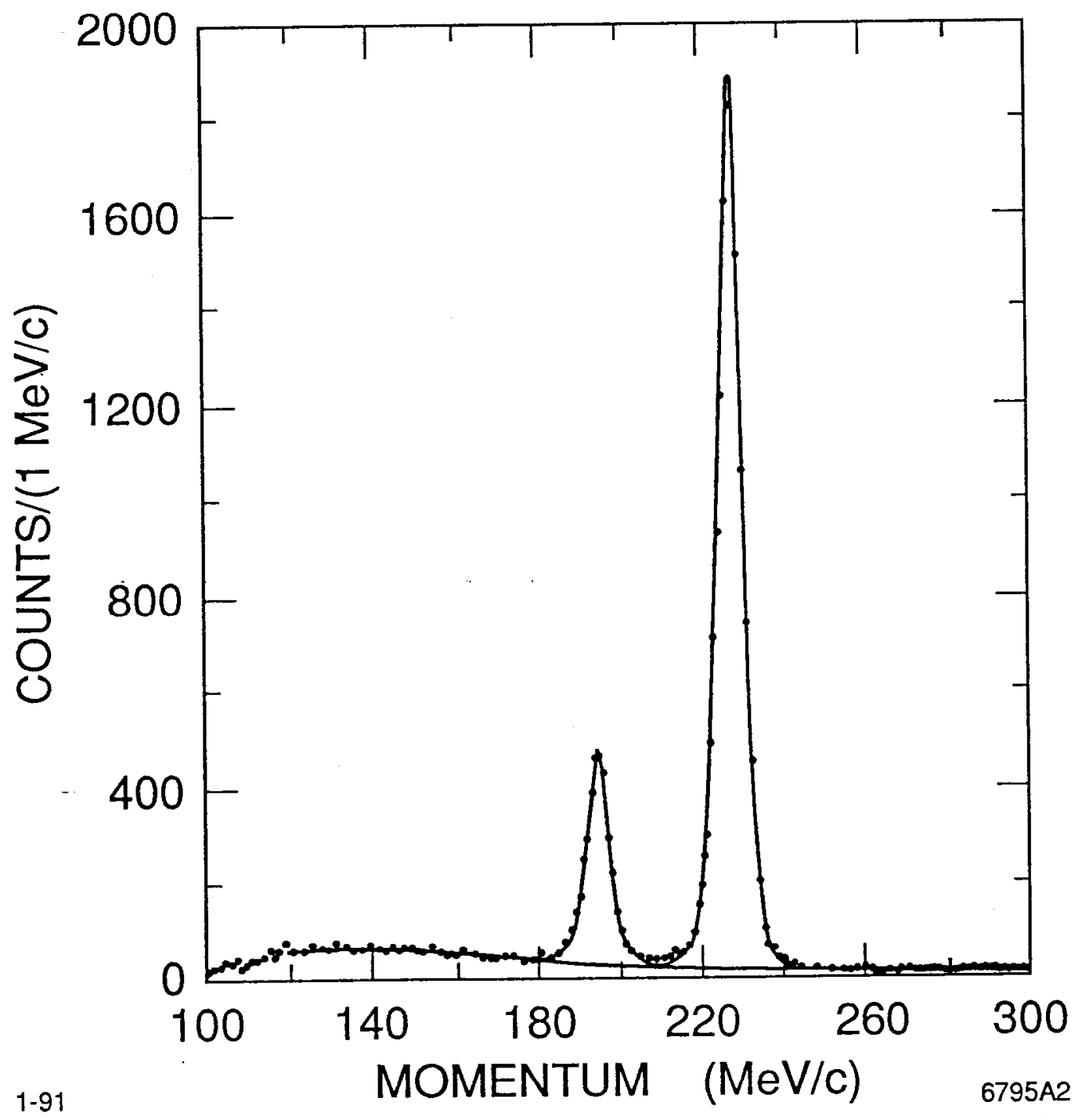

Fig. 2 


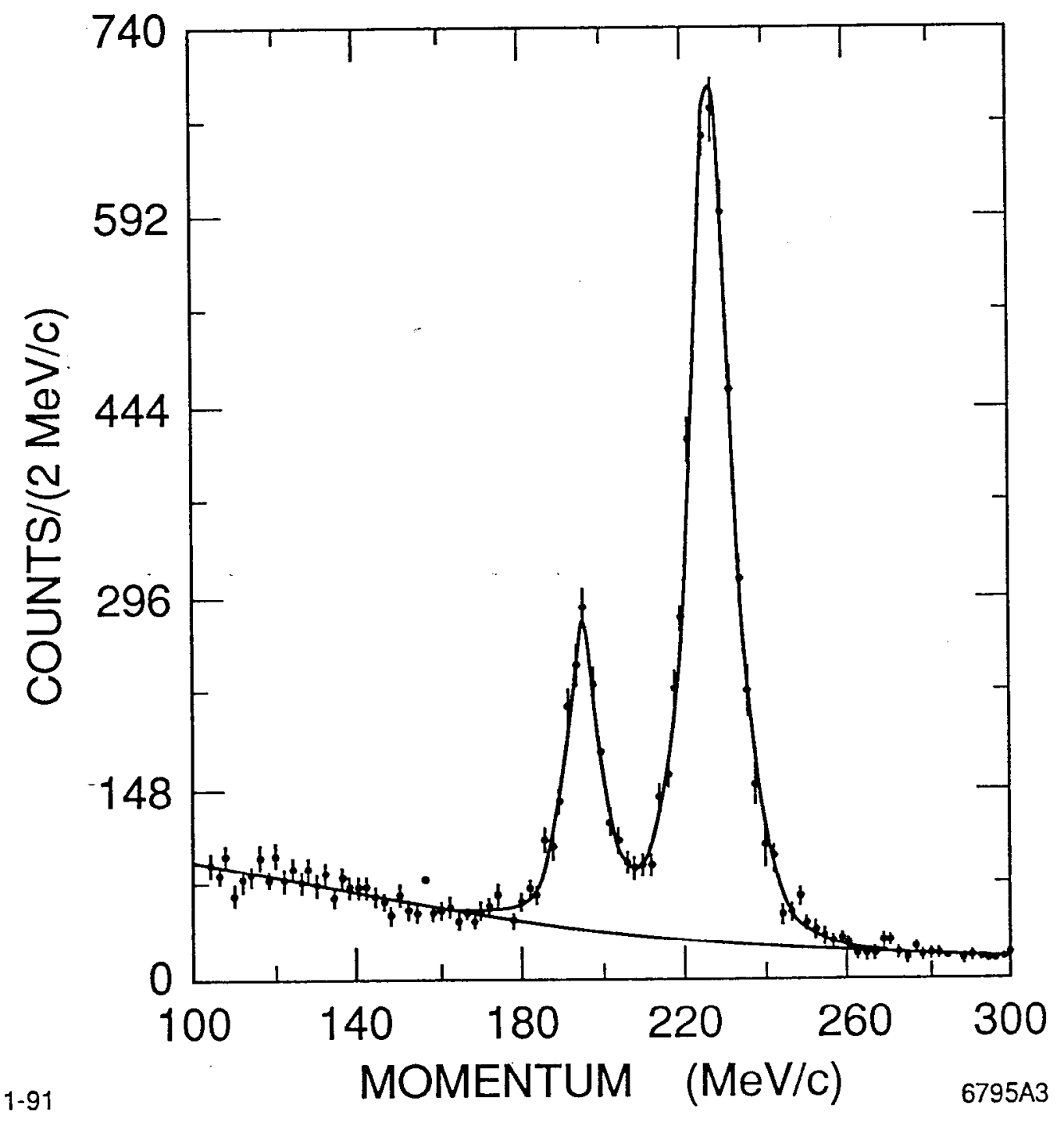

Fig. 3 